International Journal of Modern Physics B, Vol.20. No.3. (2006) pp.341-354

\title{
Transport Equations from Liouville Equations for Fractional Systems
}

\author{
Vasily E. Tarasov \\ Skobeltsyn Institute of Nuclear Physics, Moscow State University, Moscow 119992, Russia \\ E-mail: tarasov@theory.sinp.msu.ru
}

\begin{abstract}
We consider dynamical systems that are described by fractional power of coordinates and momenta. The fractional powers can be considered as a convenient way to describe systems in the fractional dimension space. For the usual space the fractional systems are non-Hamiltonian. Generalized transport equation is derived from Liouville and Bogoliubov equations for fractional systems. Fractional generalization of average values and reduced distribution functions are defined. Hydrodynamic equations for fractional systems are derived from the generalized transport equation.
\end{abstract}

PACS 05.40.-a, 02.50.Ey, 05.20.-y

Keywords: Transport equation, Liuoville equation, Bogoliubov equations, fractional integrals, hydrodynamic equations

\section{Introduction}

Integrals and derivatives of fractional order $[1,2]$ have found many applications in recent studies in physics $[3,4,5]$. The interest in fractional equations has been growing continually during the last few years because of numerous applications: kinetic theories of systems with chaotic and pseudochaotic dynamics $[6,7,8,9]$; dynamics in a complex or porous media $[10,11,12,13,14,15]$; electrodynamics $[19,20]$, dynamical systems $[21,22,23,24,25,26]$ and many others.

The new type of problems has rapidly increased interest in areas in which the fractal features of a process or the medium impose a necessity of applying the tools that are non-traditional in "regular" smooth physical equations. In many problems the real fractal structure of matter can be disregarded and the medium can be replaced by some smoothed continuous mathematical model where fractional integrals appear $[11,12,14,20]$. The order of fractional integral is equal to the fractal mass dimension of medium and in this way one can take into account the fractality of media. It was proved that integrals on net of fractals can be approximated by fractional integrals [16] and that fractional integrals can be considered as integrals over the space with fractional dimension up to a numerical factor $[17,18]$.

It is known that Bogoliubov equations can be derived from the Liouville equation and the definition of average value Refs. [29, 30, 31, 32]. In Refs. [17], the Liouville equation for fractional systems is derived from the fractional normalization condition. In [18], the first Bogoliubov equation for fractional systems is derived from the Liouville equation. It is known that hydrodynamic equations can be derived from generalized transport equation [28] which is directly derived from first Bogoliubov equation $[29,31,33]$. In this paper, the hydrodynamic equations for fractional systems are derived from the generalized Enskog transport equations.

In Sec. 2, the fractional average values and some notations are considered. In Sec. 3, we define the reduced one-particle and two-particle distribution functions. In Sec. 4, the Liouville and first fractional 
Bogoliubov equations for fractional systems are considered. In Sec. 5, we derive the fractional analog of the Enskog transport equation. In Sec. 6, the hydrodynamic equations for fractional systems are derived from the generalized transport equations. Finally, a short conclusion is given in Sec. 7 .

\section{Fractional Systems}

Let us consider a dynamical system that is described by the coordinates $\bar{q}_{k}$ and momenta $\bar{p}_{k}$ that satisfy the equations of motion:

$$
\frac{d \bar{q}_{k}}{d \bar{t}}=\frac{\bar{p}_{k}}{M}, \quad \frac{d \bar{q}_{k}}{d \bar{t}}=f_{k}(\bar{q}, \bar{p}, \bar{t}) \quad k=1, \ldots, n .
$$

Let us introduce the dimensionless variables

$$
q_{k}=\frac{\bar{q}_{k}}{q_{0}}, \quad p_{k}=\frac{\bar{p}_{k}}{p_{0}}, \quad t=\frac{\bar{t}}{t_{0}}, \quad F_{k}=\frac{f_{k}}{F_{0}},
$$

where $q_{0}$ is a characteristic scale in the configuration space; $p_{0}$ is a characteristic momentum, $F_{0}$ is a characteristic value of force, and $t_{0}$ is a typical time. Then Eq. (1) has the form

$$
\frac{d q_{k}}{d t}=\frac{p_{k}}{m}, \quad \frac{d q_{k}}{d \bar{t}}=Z F_{k}(q, p, t)
$$

where

$$
m=M q_{0} / t_{0} p_{0}, \quad Z=t_{0} F_{0} / p_{0} .
$$

are dimensionless parameters. Using the dimensionless variables $(q, p, t)$, we can generalize Eq. (2) for arbitrary powers of $q_{k}$ and $p_{k}$ :

$$
\frac{d q_{k}^{\alpha}}{d t}=\frac{p_{k}^{\alpha}}{m}, \quad \frac{d q_{k}^{\alpha}}{d \bar{t}}=Z F_{k}\left(q^{\alpha}, p^{\alpha}, t\right)
$$

where

$$
\begin{aligned}
q_{k}^{\alpha} & =\beta(q)\left(q_{k}\right)^{\alpha}=\operatorname{sgn}\left(q_{k}\right)\left|q_{k}\right|^{\alpha}, \\
p_{k}^{\alpha} & =\beta(p)\left(p_{k}\right)^{\alpha}=\operatorname{sgn}\left(p_{k}\right)\left|p_{k}\right|^{\alpha} .
\end{aligned}
$$

Here $k=1, \ldots, n$, and $\beta(x)=[\operatorname{sgn}(x)]^{\alpha-1}$. The function $\operatorname{sgn}(x)$ is equal to +1 for $x \geq 0$, and -1 for $x<0$.

A system is called a fractional system if the phase space is described by the fractional powers of coordinates (5) and momenta (6). We can describe the fractional systems in the usual phase space $(q, p)$ and in the fractional phase space $\left(q^{\alpha}, p^{\alpha}\right)$. In the second case, the equations of motion for the fractional systems are more simple. Therefore we use the fractional phase space. The fractional space is considered as a space with the fractional measure that is used in the fractional integrals.

The generalization of Hamiltonian system is described by

$$
\frac{d q_{k}^{\alpha}}{d t}=\frac{\partial H}{\partial p_{k}^{\alpha}}, \quad \frac{d p_{k}^{\alpha}}{d t}=-\frac{\partial H}{\partial q_{k}^{\alpha}},
$$

where $H$ is an analog of the Hamiltonian. Using the fractional power Poisson brackets

$$
\{A, B\}_{\alpha}=\sum_{k=1}^{n}\left(\frac{\partial A}{\partial q_{k}^{\alpha}} \frac{\partial B}{\partial p_{k}^{\alpha}}-\frac{\partial A}{\partial p_{k}^{\alpha}} \frac{\partial B}{\partial q_{k}^{\alpha}}\right),
$$


we rewrite Eq. (7) in the from

$$
\frac{d q_{k}^{\alpha}}{d t}=\left\{q_{k}^{\alpha}, H\right\}_{\alpha}, \quad \frac{d p_{k}^{\alpha}}{d t}=\left\{p_{k}^{\alpha}, H\right\}_{\alpha} .
$$

These equations describe the system in the fractional phase space $\left(q^{\alpha}, p^{\alpha}\right)$. For the usual phase space $(q, p)$, Eq. (7) has the form

$$
\frac{d q_{k}}{d t}=\frac{\left(q_{k} p_{k}\right)^{1-\alpha}}{\alpha^{2}} \frac{\partial H}{\partial p_{k}}, \quad \frac{d p_{k}}{d t}=-\frac{\left(q_{k} p_{k}\right)^{1-\alpha}}{\alpha^{2}} \frac{\partial H}{\partial q_{k}},
$$

and these systems are non-Hamiltonian systems. A classical system is called Hamiltonian if the righthand sides of the equations

$$
\frac{d q_{k}}{d t}=g_{k}(q, p), \quad \frac{d p_{k}}{d t}=f_{k}(q, p)
$$

satisfy the Helmholtz conditions [27]:

$$
\frac{\partial g_{k}}{\partial p_{l}}-\frac{\partial g_{l}}{\partial p_{k}}=0, \quad \frac{\partial g_{k}}{\partial q_{l}}-\frac{\partial f_{l}}{\partial p_{k}}=0, \quad \frac{\partial f_{k}}{\partial q_{l}}-\frac{\partial f_{l}}{\partial q_{k}}=0 .
$$

It is easy to prove these conditions are not satisfied for Eq. (10). Therefore the dymanical system (10) is a non-Hamiltonian system. The fractional phase space allows us to write Eq. (9) in the simple form (7) and describe some non-Hamiltonian systems as a Hamiltonian systems in generalized space.

If $d q_{k}^{\alpha} / d t=p_{k}^{\beta} / m$, then an analog of Hamiltonian

$$
H_{\alpha, \beta}=\sum_{k, l=1}^{n} \frac{\alpha p_{k}^{\alpha+\beta}}{m(\alpha+\beta)}+U(q) .
$$

The omega function for system (11) is defined by

$$
\Omega=\sum_{k=1}^{n}\left(\frac{\partial g_{k}}{\partial q_{k}}+\frac{\partial f_{k}}{\partial p_{k}}\right),
$$

and describes the velocity of phase volume change. If $\Omega<0$, then the system is called a dissipative system. If $\Omega \neq 0$, then the system is a generalized dissipative system. For system (10), the omega function (14) is not equal to zero, and the systems is the general dissipative system.

It is not hard to prove that Hamiltonian (13) is connected with the non-Gaussian statistics. Dissipative and non-Hamiltonian systems can have the canonical Gibbs distribution as a solution of the stationary Liouville equations [40]. Using [40], it is easy to prove that some of fractional systems can have fractional Gibbs distribution

$$
\rho(q, p)=\exp \left[\mathcal{F}-H_{\alpha, \beta}(q, p)\right] / k T,
$$

as a solution of Liouville equation for fractional systems [17].

\section{Fractional Average Values and Reduced Distributions}

\subsection{Fractional average values for configuration space}

Let us derive the fractional generalization of average value of classical observable $A(q, p)$. For configuration space, the usual average value is

$$
<A>_{1}=\int_{-\infty}^{+\infty} A(x) \rho(x) d x,
$$


and can be written

$$
<A>_{1}=\int_{-\infty}^{y} A(x) \rho(x) d x+\int_{y}^{\infty} A(x) \rho(x) d x .
$$

Using

$$
\begin{aligned}
& \left(I_{+}^{\alpha} f\right)(y)=\frac{1}{\Gamma(\alpha)} \int_{-\infty}^{y} \frac{f(x) d x}{(y-x)^{1-\alpha}} \\
& \left(I_{-}^{\alpha} f\right)(y)=\frac{1}{\Gamma(\alpha)} \int_{y}^{\infty} \frac{f(x) d x}{(x-y)^{1-\alpha}}
\end{aligned}
$$

we rewtite Eq. (17) in the form

$$
<A>_{1}=\left(I_{+}^{1} A \rho\right)(y)+\left(I_{-}^{1} A \rho\right)(y) .
$$

The fractional generalization of this equation is

$$
<A>_{\alpha}=\left(I_{+}^{\alpha} A \rho\right)(y)+\left(I_{-}^{\alpha} A \rho\right)(y) .
$$

We can rewrite Eq. (18) in the form

$$
<A>_{\alpha}=\frac{1}{2} \int_{-\infty}^{\infty}[(A \rho)(y-x)+(A \rho)(y+x)] d \mu_{\alpha}(x),
$$

where

$$
d \mu_{\alpha}(x)=\frac{|x|^{\alpha-1} d x}{\Gamma(\alpha)}=\frac{d x^{\alpha}}{\alpha \Gamma(\alpha)}, \quad x^{\alpha}=\operatorname{sgn}(x)|x|^{\alpha} .
$$

Equation (18) defines the fractional generalization of the average value for coordinate space.

\subsection{Fractional average values for phase space}

Let us introduce some notations to define the fractional average value for phase space. Tilde operators

$$
T_{x_{k}} f\left(\ldots, x_{k}, \ldots\right)=\frac{1}{2}\left(f\left(\ldots, x_{k}^{\prime}-x_{k}, \ldots\right)+f\left(\ldots, x_{k}^{\prime}+x_{k}, \ldots\right)\right)
$$

allows us to rewrite

$$
\begin{aligned}
& \frac{1}{4}\left(A\left(q^{\prime}-q, p^{\prime}-p, t\right) \rho\left(q^{\prime}-q, p^{\prime}-p, t\right)+A\left(q^{\prime}+q, p^{\prime}-p, t\right) \rho\left(q^{\prime}+q, p^{\prime}-p, t\right)+\right. \\
& \left.+A\left(q^{\prime}-q, p^{\prime}+p, t\right) \rho\left(q^{\prime}-q, p^{\prime}+p, t\right)+A\left(q^{\prime}+q, p^{\prime}+p, t\right) \rho\left(q^{\prime}+q, p^{\prime}+p, t\right)\right)
\end{aligned}
$$

in the simple form

$$
T_{q} T_{p}(A(q, p, t) \rho(q, p, t)) .
$$

For $k$ particle with coordinates $q_{k s}$ and momenta $p_{k s}$, where $s=1, \ldots, m$, we define the operator

$$
T[k]=T_{q_{k 1}} T_{p_{k 1} \ldots T_{q_{k m}}} T_{p_{k m}} .
$$

For the $n$-particle system phase space, we use

$$
T[1, \ldots, n]=T[1] \ldots T[n] .
$$

Let us define the integral operators $\hat{I}_{x_{k}}^{\alpha}$ by

$$
\hat{I}_{x_{k}}^{\alpha} f\left(x_{k}\right)=\int_{-\infty}^{+\infty} f\left(x_{k}\right) d \mu_{\alpha}\left(x_{k}\right),
$$


then Eq. (19) has the form

$$
<A>_{\alpha}=\hat{I}_{x}^{\alpha} T_{x} A(x) \rho(x) .
$$

For $k$-particle we use the operator

$$
\hat{I}^{\alpha}[k]=\hat{I}_{q_{k 1}}^{\alpha} \hat{I}_{p_{k 1}}^{\alpha} \ldots \hat{I}_{q_{k m}}^{\alpha} \hat{I}_{p_{k m}}^{\alpha}
$$

such that

$$
\hat{I}^{\alpha}[k] f\left(\mathbf{q}_{k}, \mathbf{p}_{k}\right)=\int f\left(\mathbf{q}_{k}, \mathbf{p}_{k}\right) d \mu_{\alpha}\left(\mathbf{q}_{k}, \mathbf{p}_{k}\right),
$$

where $d \mu_{\alpha}\left(\mathbf{q}_{k}, \mathbf{p}_{k}\right)$ is an elementary $2 m$-dimensional phase volume

$$
d \mu_{\alpha}\left(\mathbf{q}_{k}, \mathbf{p}_{k}\right)=(\alpha \Gamma(\alpha))^{-2 m} d q_{k 1}^{\alpha} \wedge d p_{k 1}^{\alpha} \wedge \ldots \wedge d q_{k m}^{\alpha} \wedge d p_{k m}^{\alpha} .
$$

For the $n$-particle system, we use

$$
\hat{I}^{\alpha}[1, \ldots, n]=\hat{I}^{\alpha}[1] \ldots \hat{I}^{\alpha}[n] .
$$

Using the suggested notations, we can define the fractional generalization of the average value for $n$-particle by

$$
<A>_{\alpha}=\hat{I}^{\alpha}[1, \ldots, n] T[1, \ldots, n] A \rho_{n} .
$$

In the simple case $(n=m=1)$, we have

$$
<A>_{\alpha}=\int_{-\infty}^{\infty} \int_{-\infty}^{\infty} d \mu_{\alpha}(q, p) T_{q} T_{p} A(q, p) \rho(q, p) .
$$

The fractional generalization of normalization condition [17] can be written by

$$
<1>_{\alpha}=1 \text {. }
$$

\subsection{Reduced distribution functions}

Let us consider a classical system with fixed number $n$ of identical particles. Suppose $k$ particle is described by the dimensionless generalized coordinates $q_{k s}$ and generalized momenta $p_{k s}$, where $s=1, \ldots, m$. We use the notations $\mathbf{q}_{k}=\left(q_{k 1}, \ldots, q_{k m}\right)$ and $\mathbf{p}_{k}=\left(p_{k 1}, \ldots, p_{k m}\right)$. The state of this system is described by dimensionless n-particle distribution function $\rho_{n}$ in the $2 m n$-dimensional phase space

$$
\rho_{n}(\mathbf{q}, \mathbf{p}, t)=\rho\left(\mathbf{q}_{1}, \mathbf{p}_{1}, \ldots, \mathbf{q}_{n}, \mathbf{p}_{n}, t\right) .
$$

We assume that function (25) is invariant under the permutations of identical particles [34]:

$$
\rho\left(\ldots, \mathbf{q}_{k}, \mathbf{p}_{k}, \ldots, \mathbf{q}_{l}, \mathbf{p}_{l}, \ldots, t\right)=\rho\left(\ldots, \mathbf{q}_{l}, \mathbf{p}_{l}, \ldots, \mathbf{q}_{k}, \mathbf{p}_{k}, \ldots, t\right) .
$$

Then the average values can be simplified [34]. Using the tilde distribution functions

$$
\tilde{\rho}_{n}(\mathbf{q}, \mathbf{p}, t)=T[1, \ldots, n] \rho_{n}(\mathbf{q}, \mathbf{p}, t),
$$

we define

$$
\tilde{\rho}_{1}(\mathbf{q}, \mathbf{p}, t)=\tilde{\rho}\left(\mathbf{q}_{1}, \mathbf{p}_{1}, t\right)=\hat{I}^{\alpha}[2, \ldots, n] \tilde{\rho}_{n}(\mathbf{q}, \mathbf{p}, t),
$$

which is one-particle reduced distribution function. Obviously, that $\tilde{\rho}_{1}$ satisfies the normalization condition [17]:

$$
\hat{I}^{\alpha}[1] \tilde{\rho}_{1}(\mathbf{q}, \mathbf{p}, t)=1 .
$$

Two-particle reduced distribution function $\tilde{\rho}_{2}$ is defined by the fractional integration of $\tilde{\rho}_{n}$ over all $\mathbf{q}_{k}$ and $\mathbf{p}_{k}$, except $k=1,2$ :

$$
\tilde{\rho}_{2}(\mathbf{q}, \mathbf{p}, t)=\tilde{\rho}\left(\mathbf{q}_{1}, \mathbf{p}_{1}, \mathbf{q}_{2}, \mathbf{p}_{2}, t\right)=\hat{I}^{\alpha}[3, \ldots, n] \tilde{\rho}_{n}(\mathbf{q}, \mathbf{p}, t) .
$$




\section{Liouville and Bogoliubov Equations for Fractional Systems}

Let us consider the Hamilton's equations for n-particle system in the form

$$
\frac{d q_{k s}^{\alpha}}{d t}=G_{s}^{k}\left(q^{\alpha}, p^{\alpha}\right), \quad \frac{d p_{k s}^{\alpha}}{d t}=Z F_{s}^{k}\left(q^{\alpha}, p^{\alpha}, t\right)
$$

where $Z$ is defined in Eq. (3). The evolution of $\rho_{n}$ is described by the Liouville equation [17] for fractional system

$$
\frac{d \tilde{\rho}_{n}}{d t}+\Omega_{\alpha} \tilde{\rho}_{n}=0
$$

This equation can be derived [17] from the fractional normalization condition

$$
\hat{I}^{\alpha}[1, \ldots, n] \tilde{\rho}_{n}(\mathbf{q}, \mathbf{p}, t)=1 .
$$

In Eq. (31) the derivative $d / d t$ is a total time derivative

$$
\frac{d}{d t}=\frac{\partial}{\partial t}+\sum_{k, s=1}^{n, m} \frac{d q_{k s}}{d t} \frac{\partial}{\partial q_{k s}}+\sum_{k, s=1}^{n, m} \frac{d p_{k s}}{d t} \frac{\partial}{\partial p_{k s}}
$$

that can be written for the fractional powers

$$
\frac{d}{d t}=\frac{\partial}{\partial t}+\sum_{k, s=1}^{n, m} G_{s}^{k} \frac{\partial}{\partial q_{k s}^{\alpha}}+Z \sum_{k, s=1}^{n, m} F_{s}^{k} \frac{\partial}{\partial p_{k s}^{\alpha}} .
$$

The $\alpha$-omega function is

$$
\Omega_{\alpha}=\sum_{k, s=1}^{n, m}\left(\left\{G_{s}^{k}, p_{k s}^{\alpha}\right\}_{\alpha}+Z\left\{q_{k s}^{\alpha}, F_{s}^{k}\right\}_{\alpha}\right)
$$

where

$$
\{A, B\}_{\alpha}=\sum_{k, s=1}^{n, m}\left(\frac{\partial A}{\partial q_{k s}^{\alpha}} \frac{\partial B}{\partial p_{k s}^{\alpha}}-\frac{\partial A}{\partial p_{k s}^{\alpha}} \frac{\partial B}{\partial q_{k s}^{\alpha}}\right) .
$$

Using Eqs. (34) and (33), we get Eq. (31) in the form

$$
\frac{\partial \tilde{\rho}_{n}}{\partial t}=\Lambda_{n} \tilde{\rho}_{n}
$$

where $\Lambda_{n}$ is Liouville operator:

$$
\Lambda_{n} \tilde{\rho}_{n}=-\sum_{k, s=1}^{n, m}\left(\frac{\partial\left(G_{s}^{k} \tilde{\rho}_{n}\right)}{\partial q_{k s}^{\alpha}}+Z \frac{\partial\left(F_{s}^{k} \tilde{\rho}_{n}\right)}{\partial p_{k s}^{\alpha}}\right) .
$$

The Bogoliubov equations $[29,30,31,32]$ describe the evolution of the reduced distribution functions, and can be derived from the Liouville equation. In Ref. [18], we derive the first fractional Bogoliubov equation from Eq. (36) :

$$
\frac{\partial \tilde{\rho}_{1}}{\partial t}+\sum_{s=1}^{m} \frac{\partial\left(G_{s}^{1} \tilde{\rho}_{1}\right)}{\partial q_{1 s}^{\alpha}}+Z \sum_{s=1}^{m} \frac{\partial\left(F_{s}^{1 e} \tilde{\rho}_{1}\right)}{\partial p_{1 s}^{\alpha}}=(n-1) Z I\left(\tilde{\rho}_{2}\right) .
$$

Here, $I\left(\tilde{\rho}_{2}\right)$ is a term with two-particle reduced distribution function,

$$
I\left(\tilde{\rho}_{2}\right)=-\sum_{s=1}^{m} \frac{\partial}{\partial p_{1 s}^{\alpha}} \hat{I}^{\alpha}[2] F_{s}^{12} \tilde{\rho}_{2}
$$


Equation (38) is called a first Bogoliubov equation for fractional systems.

The physical meaning of the term $I\left(\tilde{\rho}_{2}\right)$ is following: The term $I\left(\tilde{\rho}_{2}\right) d \mu_{\alpha}(\mathbf{q}, \mathbf{p})$ is a velocity of particle number change in $4 m$-dimensional elementary phase volume $d \mu_{\alpha}\left(\mathbf{q}_{1}, \mathbf{p}_{2}, \mathbf{q}_{2}, \mathbf{p}_{2}\right)$. This change is caused by the interactions between particles. If $\alpha=1$, then we have the first Bogoliubov equation for non-Hamiltonian systems.

\section{Transport Equation for Fractional Systems}

Let us define the coordinate distribution (the density of number of particles) by the equation

$$
n(\mathbf{q}, t)=\hat{I}^{\alpha}[\mathbf{p}] \tilde{\rho}_{1}(\mathbf{q}, \mathbf{p}, t),
$$

where $\hat{I}[\mathbf{p}]$ is a fractional integration over the momenta

$$
\hat{I}^{\alpha}[\mathbf{p}]=\prod_{s=1}^{m} \hat{I}_{p_{s}}^{\alpha}=\hat{I}_{p_{1}}^{\alpha} \ldots \hat{I}_{p_{m}}^{\alpha} .
$$

We can define the local mean values by

$$
\langle A\rangle_{p, \alpha}=\langle A\rangle_{p, \alpha}(\mathbf{q}, t)=\frac{1}{n(\mathbf{q}, t)} \hat{I}^{\alpha}[\mathbf{p}] A(\mathbf{q}, \mathbf{p}) \tilde{\rho}_{1}(\mathbf{q}, \mathbf{p}, t) .
$$

In the general case,

$$
\langle A\rangle_{p, \alpha} \neq<A>_{\alpha},
$$

and $\langle 1\rangle_{p, \alpha}=1$. The fractional average value $\langle A\rangle_{\alpha}$ is connected with the mean value $\langle A\rangle_{p, \alpha}$ by

$$
<A>_{\alpha}=\hat{I}^{\alpha}[\mathbf{q}] n(\mathbf{q}, t)\langle A\rangle_{p, \alpha},
$$

where $\hat{I}[\mathbf{q}]$ is a fractional integration over the coordinates $\mathbf{q}=\left(q_{1}, \ldots, q_{m}\right)$.

Fractional analog of mean local velocity is

$$
V_{s}(\mathbf{q}, t)=\frac{1}{n(\mathbf{q}, t)} \hat{I}^{\alpha}[\mathbf{p}] G_{s}(\mathbf{q}, \mathbf{p}) \rho_{1}(\mathbf{q}, \mathbf{p}, t)
$$

i.e.

$$
V_{s}(\mathbf{q}, t)=\left\langle G_{s}\right\rangle_{p, \alpha}
$$

where $G_{s}=G_{s}^{1}(\mathbf{q}, \mathbf{p})$ is defined by (30). We can consider $G_{s}$ as a fractional generalization of the velocity that has the form

$$
G_{s}=\frac{p_{s}^{\alpha}}{M}
$$

For the fractional generalization of kinetic energy of relative motion

$$
\sum_{s=1}^{m} \frac{M}{2}\left(p_{s}^{\alpha}-V_{s}\right)^{2}
$$

we define density of this energy by

$$
E(\mathbf{q}, t)=\frac{M}{2} \hat{I}^{\alpha}[\mathbf{p}](\mathbf{G}-\mathbf{V})^{2} \rho_{1}(\mathbf{q}, \mathbf{p}, t),
$$


where $\mathbf{G}=\mathbf{p}^{\alpha} / m$, and $\mathbf{V}=\mathbf{V}(\mathbf{q}, t)$. The local temperature $T(\mathbf{q}, t)$ is defined by the mean kinetic energy of relative motion:

$$
T(\mathbf{q}, t)=\frac{2 E(\mathbf{q}, t)}{3 k_{B} n(\mathbf{q}, t)} .
$$

To derive Enskog transport equation for fractional systems, we multiply both sides of (38) by the observable $A(\mathbf{p})$, and integrate with respect to momenta. The first and second terms of left hand side of Eq. (38) are transformed by

$$
\begin{gathered}
\hat{I}[\mathbf{p}] A \frac{\partial \tilde{\rho}_{1}}{\partial t}=\frac{\partial}{\partial t} \hat{I}[\mathbf{p}] A \tilde{\rho}_{1}=\frac{\partial}{\partial t} n(\mathbf{q}, t)\langle A\rangle_{p, \alpha}(\mathbf{q}, t), \\
\hat{I}[\mathbf{p}] A \frac{\partial\left(G_{s} \tilde{\rho}_{1}\right)}{\partial q_{s}^{\alpha}}=\frac{\partial}{\partial q_{s}^{\alpha}} \hat{I}[\mathbf{p}] A G_{s} \tilde{\rho}_{1}=\frac{\partial}{\partial q_{s}^{\alpha}} n(\mathbf{q}, t)\left\langle A G_{s}\right\rangle_{p, \alpha} .
\end{gathered}
$$

Integrating by part the third term of Eq. (38) and using the boundary condition

$$
\lim _{p \rightarrow \pm \infty} \tilde{\rho}_{1}(\mathbf{q}, \mathbf{p}, t)=0
$$

we get

$$
\begin{aligned}
& \hat{I}[\mathbf{p}] A \frac{\partial\left(F_{s} \tilde{\rho}_{1}\right)}{\partial p_{s}^{\alpha}}=\hat{I}[\mathbf{p}] \frac{\partial}{\partial p_{s}^{\alpha}} A F_{s} \tilde{\rho}_{1}-\hat{I}[\mathbf{p}] F_{s} \tilde{\rho}_{1} \frac{\partial A}{\partial p_{s}^{\alpha}}= \\
= & \left(A F_{s} \tilde{\rho}_{1}\right)_{-\infty}^{+\infty}-\hat{I}[\mathbf{p}] F_{s} \tilde{\rho}_{1} \frac{\partial A}{\partial p_{s}^{\alpha}}=-n(\mathbf{q}, t)\left\langle F_{s} \frac{\partial A}{\partial p_{s}^{\alpha}}\right\rangle_{p, \alpha} .
\end{aligned}
$$

Then we use the usual assumption

$$
\hat{I}[\mathbf{p}] A(\mathbf{p}) I\left(\tilde{\rho}_{2}\right)=0 .
$$

for $A=M, A=p_{s}^{\alpha}$ and $A=\mathbf{p}^{2 \alpha}$.

Finally, we obtain the Enskog transport equation for fractional systems:

$$
\frac{\partial}{\partial t}\left(n(\mathbf{q}, t)\langle A\rangle_{p, \alpha}\right)+\frac{\partial}{\partial \mathbf{q}^{\alpha}}\left(n(\mathbf{q}, t)\langle A \mathbf{G}\rangle_{p, \alpha}\right)=n(\mathbf{q}, t) Z\left\langle\mathbf{F} \frac{\partial A}{\partial \mathbf{p}^{\alpha}}\right\rangle_{p, \alpha},
$$

where

$$
\mathbf{F} \frac{\partial}{\partial \mathbf{p}^{\alpha}}=\sum_{s=1}^{m} F_{s} \frac{\partial}{\partial p_{s}^{\alpha}}, \quad \frac{\partial}{\partial \mathbf{q}^{\alpha}}\left(n(\mathbf{q}, t)\langle A \mathbf{G}\rangle_{p, \alpha}\right)=\sum_{s=1}^{m} \frac{\partial}{\partial q_{s}^{\alpha}}\left(n(\mathbf{q}, t)\left\langle A G_{s}\right\rangle_{p, \alpha}\right) .
$$

\section{Hydrodynamic Equation for Fractional Systems}

Let us consider the special cases of transport equation (54) for

$$
A=M, \quad A=p_{s}^{\alpha}=M G_{s}, \quad A=\frac{\mathbf{p}^{2 \alpha}}{2 M} .
$$

If we use $A=M$, then (54) gives

$$
\frac{\partial}{\partial t} \tilde{\rho}_{M}(\mathbf{q}, t)+\frac{\partial}{\partial q_{s}^{\alpha}} \tilde{\rho}_{M}(\mathbf{q}, t)\left\langle G_{s}\right\rangle_{p, \alpha}(\mathbf{q}, t)=0,
$$

where $\rho_{M}$ is mass density

$$
\tilde{\rho}_{M}(\mathbf{q}, t)=M n(\mathbf{q}, t)
$$


For $A=p_{s}^{\alpha}=M G_{s}$, we obtain

$$
\frac{\partial}{\partial t} \tilde{\rho}_{M} V_{l}(\mathbf{q}, t)+\frac{\partial}{\partial q_{s}^{\alpha}} \tilde{\rho}_{M}\left\langle G_{s} G_{l}\right\rangle_{p, \alpha}=\tilde{\rho}_{M}(\mathbf{q}, t) Z\left\langle F_{l}\right\rangle_{p, \alpha},
$$

where we use Eqs. (46), (56), and the relation

$$
\frac{\partial G_{l}}{\partial p_{s}^{\alpha}}=M \delta_{l s}
$$

For $A=\mathbf{p}^{2 \alpha} / 2 M$, we get

$$
\frac{\partial}{\partial t} \tilde{\rho}_{M}(\mathbf{q}, t)\left\langle\frac{\mathbf{p}^{2 \alpha}}{2 M^{2}}\right\rangle_{p, \alpha}+\frac{\partial}{\partial q_{s}^{\alpha}} \tilde{\rho}_{M}(\mathbf{q}, t)\left\langle\frac{1}{2} G_{l}^{2} G_{s}\right\rangle_{p, \alpha}=\tilde{\rho}_{M}(\mathbf{q}, t) Z\left\langle F_{s} G_{s}\right\rangle_{p, \alpha} .
$$

Here $\left\langle F_{s} G_{s}\right\rangle_{p, \alpha}$ is a local mean value.

Let us define the deviation of velocity from its mean value by

$$
C_{s}(\mathbf{q}, t)=G_{s}-V_{s}(\mathbf{q}, t)=G_{s}-\left\langle G_{s}\right\rangle_{p, \alpha}
$$

Substituting $G_{s}=V_{s}+C_{s}$ in the kinetic energy tensor $\left\langle G_{s} G_{l}\right\rangle_{p, \alpha}$, we get

$$
\left\langle G_{s} G_{l}\right\rangle_{p, \alpha}=V_{s} V_{s}-\left\langle C_{s} C_{l}\right\rangle_{p, \alpha},
$$

where we use $\left\langle C_{s}\right\rangle_{p, \alpha}=0$. From Eq. (60), we have

$$
\left\langle\frac{\mathbf{p}^{2 \alpha}}{2 M}\right\rangle_{p, \alpha}=\frac{M \mathbf{V}^{2}}{2}+\frac{M \mathbf{C}^{2}}{2} .
$$

The tensor of internal stress

$$
P_{s l}=\left\langle C_{s} C_{l}\right\rangle_{p, \alpha}
$$

can be represented as the sum

$$
P_{s l}(\mathbf{q}, t)=\delta_{s l} P(\mathbf{q}, t)+\pi_{s l}(\mathbf{q}, t), \quad \pi_{s s}(\mathbf{q}, t)=0,
$$

where $\pi_{s l}(\mathbf{q}, t)$ is the tensor of viscous stress. Then

$$
\begin{gathered}
\frac{\rho_{M}}{2}\left\langle G_{l}^{2} G_{s}\right\rangle_{p, \alpha}=\frac{\rho_{M}}{2}\left\langle\left(V_{l}+C_{l}\right)^{2}\left(V_{s}+C_{s}\right)\right\rangle_{p, \alpha}= \\
=\frac{\rho_{M}}{2}\left\langle\left(V_{l}^{2}+C_{l}^{2}+2 V_{l} C_{l}\right)\left(V_{s}+C_{s}\right)\right\rangle_{p, \alpha}= \\
=\frac{\rho_{M}}{2}\left(V_{s}^{2}+\left\langle C_{l}^{2}\right\rangle_{p, \alpha}\right) V_{l}+2 V_{l} P_{l s}+\frac{\rho_{M}}{2}\left\langle C_{l}^{2} C_{s}\right\rangle_{p, \alpha} .
\end{gathered}
$$

Substituting Eq. (62) in Eq. (57), and substituting Eqs. (61), (63) in Eq. (58), we get the equations for the functions $\rho_{M}(\mathbf{q}, t), V_{s}(\mathbf{q}, t), E(\mathbf{q}, t)$. From (55), we get the continuity equation for fractional systems $[17,18]$ :

$$
\frac{\partial}{\partial t} \tilde{\rho}_{M}+\frac{\partial}{\partial q_{s}^{\alpha}} \tilde{\rho}_{M} V_{s}=0
$$

which may be regarded as the equation of balance of "fractional matter". This matter can be described by fractional systems (30). In addition to $\rho_{M}$, the continuity equation (64) includes the density of momentum $\rho_{M} \mathbf{V}$. To obtain the equation for the density of momentum, we multiplied first fractional Bogoliubov equation by $\mathbf{p}^{\alpha}$, and use fractional integration over $\mathbf{p}$. Taking advantage of the assumption 
(53) and the boundary condition (51), we get the equation for the components of the vector of density of momentum

$$
\frac{\partial}{\partial t} \tilde{\rho}_{M} V_{l}+\frac{\partial}{\partial q_{s}^{\alpha}}\left(\tilde{\rho}_{M} V_{s} V_{l}+P_{s l}\right)=f_{l}
$$

where $f_{l}=f_{l}(\mathbf{q}, t)=Z\left\langle F_{s}\right\rangle_{p, \alpha}$.

Finally, to write down the equation of balance of fractional kinetic energy density, we multiplied first fractional Bogoliubov equation by $\mathbf{p}^{\alpha}$, and use fractional integrated with respect to $\mathbf{p}$. Taking advantage of the assumption (53) and the boundary condition (51), we come to the equation for the density of kinetic energy of fractional systems:

$$
\frac{\partial}{\partial t}\left(\frac{1}{2} \tilde{\rho}_{M} V^{2}+E\right)+\frac{\partial}{\partial q_{s}^{\alpha}}\left(V_{s}\left[\frac{\tilde{\rho}_{M} V^{2}}{2}+E\right]+P_{s l} V_{l}+Q_{s}\right)=f_{s} V_{s}
$$

where

$$
Q_{s}=\frac{\rho_{M}}{2}\left\langle C_{l}^{2} C_{s}\right\rangle_{p, \alpha}
$$

Equations (64), (65), and (66) are the hydrodynamic equations for fractional systems. Obviously, the set of this five equations is not closed. If we have $\pi_{s l}=0$ and $Q_{s}=0$, then these equations are

$$
\begin{gathered}
\frac{\partial}{\partial t} \tilde{\rho}_{M}+\frac{\partial}{\partial q_{s}^{\alpha}} \tilde{\rho}_{M}\left\langle G_{s}\right\rangle_{p, \alpha}=0 \\
\frac{\partial}{\partial t} \tilde{\rho}_{M} V_{l}+\frac{\partial}{\partial q_{s}^{\alpha}}\left(\tilde{\rho}_{M} V_{s} V_{l}\right)=-\frac{\partial P}{\partial q_{l}^{\alpha}}+f_{l} \\
\frac{\partial}{\partial t}\left(\frac{1}{2} \tilde{\rho}_{M} V^{2}+E\right)+\frac{\partial}{\partial q_{s}^{\alpha}}\left(V_{s}\left[\frac{\tilde{\rho}_{M} V^{2}}{2}+E+P\right]\right)=f_{s} V_{s}
\end{gathered}
$$

and the set of equations is closed.

\section{Conclusion}

In this paper the hydrodynamic equations for fractional systems are derived. In order to derive these equations, we use the first Bogoliubov equation for fractional systems [18]. Then we define the fractional generalization of the average values and the reduced distribution functions. The Enskog equation for fractional systems is considered. Hydrodynamic equations (64), (65), and (66) can be considered as equations in the fractional space $[17,18]$ or for systems with non-Gaussian statistics $[17,18]$.

Dissipative and non-Hamiltonian systems can have stationary states of the Hamiltonian systems [39]. Classical dissipative systems can have canonical Gibbs distribution as solutions of Liouville equations for the dissipative systems $[40,41,14]$. Using the methods $[40,14]$ it is easy to find solutions for the Bogoliubov equations for fractional systems. Suggested Bogoliubov equation allows to formulate the dynamics for fractional generalization of quantum dissipative systems by methods suggested in $[36,37,38]$.

\section{References}

[1] K.B. Oldham, J. Spanier, The Fractional Calculus (Academic Press, New York, 1974)

[2] S.G. Samko, A.A. Kilbas, O.I. Marichev, Fractional Integrals and Derivatives Theory and Applications, (Gordon and Breach, New York, 1993). 
[3] Fractals and Fractional Calculus in Continuum Mechanics, Editors: A. Carpinteri and F. Mainardi. (Springer, Wien and New York 1997)

[4] B.J. West, M. Bologna, P. Grigolini, Physics of Fractal Operators (Springer, New York, 2003)

[5] R. Hilfer (Ed.), Applications of Fractional Calculus in Physics, (World Scientific, Singapore, 2000).

[6] G.M. Zaslavsky, Phys. Rep. 371, 461-580 (2001).

[7] A.I. Saichev, G.M. Zaslavsky, Chaos 7, 753-764 (1997).

[8] G.M. Zaslavsky, Hamiltonian Chaos and Fractional Dynamics, (Oxford University Press, Oxford, 2005).

[9] G.M. Zaslavsky, M. Edelman, Chaos 11, 295-305 (2001).

[10] R. Nigmatullin, Phys. Stat. Solidi B 133, 425-430 (1986).

[11] V.E. Tarasov, Phys. Lett. A 336, 167-174 (2005); E-print cont-mat/0506137.

[12] V.E. Tarasov, Phys. Lett. A 341, 467-472 (2005); E-print physics/0602121

[13] V.E. Tarasov, G.M. Zaslavsky, Physica A 354, 246-261 (2005); E-print physics/0511144.

[14] V.E. Tarasov, Ann. Phys. 318, 286-307 (2005); E-print physics/0602096.

[15] V.E. Tarasov, Chaos 15, 023102 (2005); E-print nlin.CD/0602029.

[16] F.Y. Ren, J.R. Liang, X.T. Wang, W.Y. Qiu, Chaos, Solitons and Fractals 16, 107-117 (2003).

[17] V.E. Tarasov, Chaos 14, 123-127 (2004); E-print nlin.CD/0312044.

[18] V.E. Tarasov, Phys. Rev. E 71, 011102 (2005); E-print cond-mat/0505720.

[19] B.A. Carreras, V.E. Lynch, G.M. Zaslavsky, Physics of Plasmas 8, 5096-5103 (2001).

[20] V.E. Tarasov, Physics of Plasmas 12, 082106 (2005).

[21] R. Gorenflo, F. Mainardi, Fractional Calculus: Integral and Differential Equations of Fractional Order, in Fractals and Fractional Calculus in Continuum Mechanics, Editors: A. Carpinteri and F. Mainardi. (Springer, Wien and New York 1997), pp. 223-276.

[22] R. Gorenflo, F. Mainardi, J. Comput. Appl. Math. 118, 283-299 (2000).

[23] F. Mainardi, Chaos, Solitons and Fractals 7, 1461-1477 (1996).

[24] V.E. Tarasov, J. Phys. A 38, 5929-5943 (2005); E-print math.DS/0602208.

[25] V.E. Tarasov, Lett. Math. Phys. 73, 49-58 (2005); E-print nlin.CD/0604007.

[26] A.A. Stanislavsky, Phys. Rev. E 70, 051103 (2004).

[27] V.E. Tarasov, Theor. Phys. 110, 57-67 (1997).

[28] D. Enskog, Arkiv. Mat., Astron. Fysik A 21, No.13., 1 (1928).

[29] N.N. Bogoliubov, Selected Works. Vol.2. (Naukova dumka, Kiev, 1970) pp.258-276.

[30] N. Bogolybow, J. Phys. USSR 10, 265 (1946)

[31] K.P. Gurov, Foundation of Kinetic Theory. Method of N.N. Bogoliubov. (Nauka, Moscow, 1966) Section I.9.

[32] D.Ya. Petrina, V.I. Gerasimenko, P.V. Malishev, Mathematical Basis of Classical Statistical Mechanics. (Naukova dumka, Kiev, 1985)

[33] S. Chapman, T. Cowling, The Mathematical Theory of Nonuniform Gases (Cambridge, 1939).

[34] N.N. Bogoliubov, N.N. Bogoliubov (Jr.), Introduction to Quantum Statistic Mechanics (Nauka, Moscow, 1984) Section 1.4.

[35] V.E. Tarasov, Mathematical Introduction to Quantum Mechanics (MAI, Moscow, 2000).

[36] V.E. Tarasov, Phys. Lett. A 288, 173-182 (2001); E-print quant-ph/0311159.

[37] V.E. Tarasov, Moscow Univ. Phys. Bull. 56/6, 5-10 (2001).

[38] V.E. Tarasov, J. Phys. A, 37, 3241-3257 (2004).

[39] V.E. Tarasov, Phys. Rev. E. 66, 056116 (2002); E-print quant-ph/0311177.

[40] V.E. Tarasov, Mod. Phys. Lett. B 17, 1219-1226 (2003); E-print cond-mat/0311536.

[41] V.E. Tarasov, J. Phys. A 38, 2145-2155 (2005) E-print math.DS/0602433.

[42] V.E. Tarasov, Ann. Phys. 316, 393-413 (2005); E-print cond-mat/0602409. 